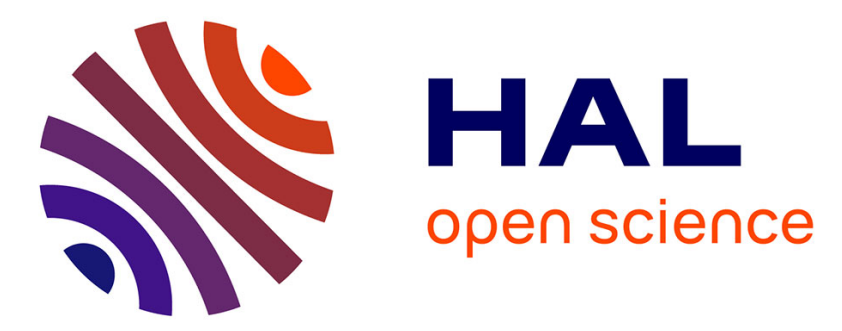

\title{
Using Comanipulation with Active Force Feedback to Undistort Stiffness Perception in Laparoscopy
}

François Schmitt, Josue Sulub, Ignacio Avellino, Jimmy da Silva, Laurent Barbé, Olivier Piccin, Bernard Bayle, Guillaume Morel

\section{- To cite this version:}

François Schmitt, Josue Sulub, Ignacio Avellino, Jimmy da Silva, Laurent Barbé, et al.. Using Comanipulation with Active Force Feedback to Undistort Stiffness Perception in Laparoscopy. 2019 International Conference on Robotics and Automation (ICRA), May 2019, Montréal, Canada. 10.1109/ICRA.2019.8793662 . hal-02343485

\section{HAL Id: hal-02343485 \\ https://hal.science/hal-02343485}

Submitted on 2 Nov 2019

HAL is a multi-disciplinary open access archive for the deposit and dissemination of scientific research documents, whether they are published or not. The documents may come from teaching and research institutions in France or abroad, or from public or private research centers.
L'archive ouverte pluridisciplinaire HAL, est destinée au dépôt et à la diffusion de documents scientifiques de niveau recherche, publiés ou non, émanant des établissements d'enseignement et de recherche français ou étrangers, des laboratoires publics ou privés. 


\title{
Using comanipulation with active force feedback to undistort stiffness perception in laparoscopy
}

\author{
François Schmitt ${ }^{\dagger}$, Josue Sulub ${ }^{\star}$, Ignacio Avellino ${ }^{\star}$, Jimmy Da Silva ${ }^{\star}$, \\ Laurent Barbé ${ }^{\dagger}$, Olivier Piccin ${ }^{\dagger}$, Bernard Bayle ${ }^{\dagger}$, and Guillaume Morel ${ }^{\star}$
}

\begin{abstract}
Surgeons performing laparoscopic surgery experience distortion when perceiving the stiffness of a patient's tissues. This is due to the lever effect induced by the introduction of instruments in their patient's body through a fulcrum. To address this problem, we propose to use the comanipulation paradigm. A robotic device is connected to the handle of the instrument while simultaneously being held by the surgeon. This device applies a force on the handle that reflects the force measured at the tool tip, with a gain that depends on the lever ratio. The implementation of this method is presented on an experimental setup and a preliminary assessment experiment is presented.
\end{abstract}

\section{INTRODUCTION}

Minimally Invasive Surgery (MIS), and more specifically Laparoscopic Surgery have been increasingly adopted since the 1980's. Their success in the surgery of the digestive system, gynecology and urology are tied to the lower risk of infection, the shortening of both the surgery and the recovery time, and also to the reduced post-operative scars. However MIS also introduces major drawbacks compared to open surgery. The use of long instruments and a camera restrict tool movement, resulting in the loss of dexterity, the deterioration of haptic an visual feedback, and the adoption of bad postures for surgeons.

Another shortcoming of MIS is that the elongated tools disrupt the perception of the forces applied at the tool tip. This is of particular concern as previous work point out the importance of force feedback to improve the safety of surgical interventions [1] as well as the role of haptic feedback in skill learning [2].

Evaluating tissue stiffness by palpation is however made difficult by the fulcrum effect [3]. This effect is caused by the insertion of the tool through the trocar, a tubular device placed at the opening in the abdominal wall and constraining the tool motion. The fulcrum point inverts the movement between the proximal handle, manipulated by the physician, and the distal instrument tip, in contact with the organs. Furthermore, depending on the tool insertion depth, the displacements and the transmitted forces are amplified between both ends. The combination of these effects generates a distortion between the stiffness perceived by the surgeon and the actual stiffness of the manipulated

$\dagger$ Université de Strasbourg, CNRS, ICube, F-67000 Strasbourg, France. O.P. is also with INSA Strasbourg, F-67000 Strasbourg, France * Sorbonne Université, CNRS, INSERM, ISIR-Agathe, F-75005, Paris, France - Corresponding E-mail: f.schmitt@unistra.fr tissues [4]. Indeed, from a perception standpoint, the surgeon evaluates the stiffness of the environment by integrating multiple stimuli, including haptic and visual cues [5]-[7]. However, the stiffness interpretation process from multiple stimulations is still being investigated [8], [9].

Robotic assistance can address both issues of force/stiffness perception and dexterity. One way to counter the fulcrum effect consists in using a tele-operation device that mechanically uncouples the movements of the physician and those of the tool. By the mean of position and force bilateral control, transparent force/displacement restitution is possible, which theoretically should lead to improved stiffness perception. However, though several tele-operated laparoscopic systems have already been proposed in the literature [10]-[14], to our knowledge, the question of stiffness restitution or compensation is yet to be addressed.

Comanipulation is an alternative to tele-operation for assistance to gesture. In this paradigm, a robot and a surgeon simultaneously manipulate an instrument. The robot can be programmed to apply forces on the handle in order to provide various functions, such as tremor filtering or instrument holding. Comanipulation has been used in the context of laparoscopic surgery: Zemiti et al., [15], proposed a compact collaborative manipulator that applies forces on a laparoscopic instrument to remove the friction forces at the trocar, although this has no influence on the fulcrum effect and thus no impact on the tissue stiffness perception.

In this paper, we focus on compensating the fulcrum effect using a comanipulation robot. The robot, called Achille, was developed in our research group. Technical details on the robot can be found in [16].

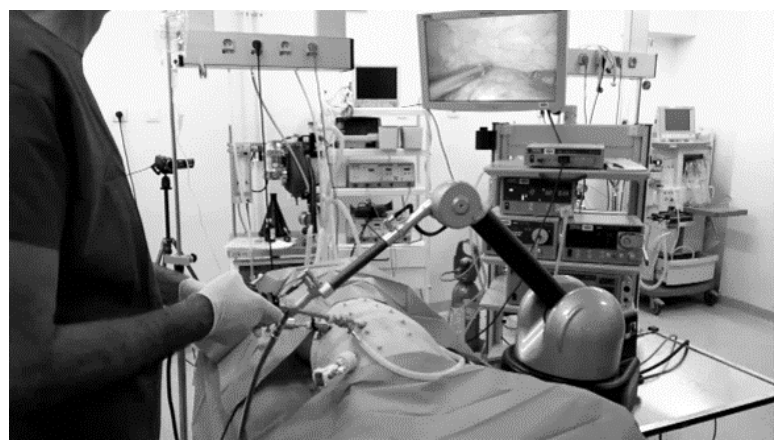

Fig. 1. Achille, a comanipulator to assist laparoscopic surgery. 


\section{Modelling}

\section{A. The lever effect}

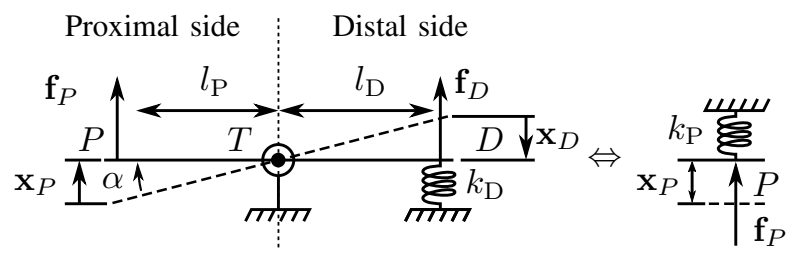

Fig. 2. Linearized lever model.

The fulcrum effect can be simply explained in a planar problem. Consider the one-degree-of-freedom (DoF) tool shown in Fig. 2 pressing on an elastic tissue. The tool can rotate about the trocar point $T$. Point $D$ (resp. $P$ ) denotes the distal (resp. proximal) extremity of the tool, and $l_{\mathrm{D}}$ (resp. $\left.l_{\mathrm{P}}\right)$ the distance between this point and $T$.

The user manipulates the tool, producing a small displacement $\mathbf{x}_{P}$ at the proximal side. The tool rotates about $T$ by a small angle $\alpha$, generating a small displacement $\mathbf{x}_{D}$ at its distal tip. The displacement of the tool then compresses the tissue, here modeled as a linear spring of stiffness $k_{\mathrm{D}}$, creating the force $\mathbf{f}_{D}$. Finally, considering the tool is in static equilibrium, the user applies a force $\mathbf{f}_{P}$ to balance the system. As the force applied by the user on the tool depends on the displacement $\mathbf{x}_{P}$, the system composed by the tool and the organ behaves as an equivalent spring of stiffness $k_{\mathrm{P}}$ at $P$.

To determine this stiffness, a geometrical evaluation of the system gives the relation between the displacements $l_{\mathrm{P}}\left\|\mathbf{x}_{D}\right\|=l_{\mathrm{D}}\left\|\mathbf{x}_{P}\right\|$. In static equilibrium, we can derive $k_{\mathrm{P}}=\mathbf{f}_{P} /\left\|\mathbf{x}_{P}\right\|$, and consequently, the stiffness equivalent to the system coupled with the environment becomes

$$
k_{\mathrm{P}}=\left(\frac{l_{\mathrm{D}}}{l_{\mathrm{P}}}\right)^{2} k_{\mathrm{D}} \text {. }
$$

This means that the stiffness experienced by the user is dependent on the square of the lever ratio, because both the displacement and the force are amplified in a lever system.

\section{B. Forces applied to a comanipulated laparoscopic tool}

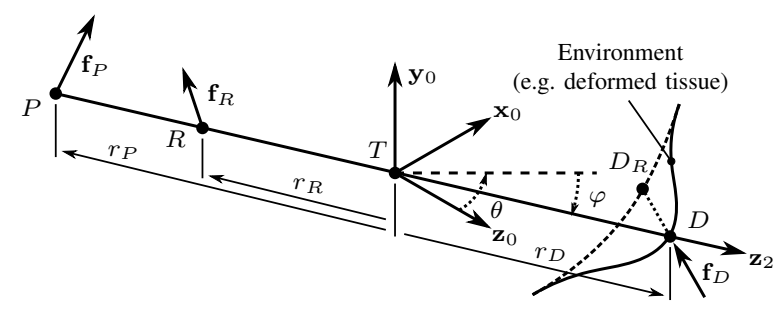

Fig. 3. Schematic representation of the laparoscopic tool and its parametrization.

Generalizing the previous example to multiple DoF, we represent the tool as a rigid bar, depicted in Fig. 3. The bar is able to rotate about Point $T$, representing the trocar, and to translate along the penetration axis $\left(T, \mathbf{z}_{2}\right)$. Three reference frames are defined. Their origins $O_{0}, O_{1}$ and $O_{2}$ are all coincident at the trocar point $T$, assumed stationary, and the rotations between reference frames are defined as follows:

$$
B_{0} \stackrel{R\left(\theta, \mathbf{y}_{0}\right)}{\longrightarrow} B_{1} \stackrel{R\left(\varphi, \mathbf{x}_{1}\right)}{\longrightarrow} B_{2} .
$$

Moreover, three points of interest are defined on the tool:

1) $P$, the proximal end of the tool, manipulated by the user,

2) $D$, the distal end of the tool, interacting with the environment, and

3) $R$, an anchoring point where the tool is attached to a robot able to generate controlled forces.

In order to simplify notation, we introduce the point $M$ acting as a placeholder for any of those points where similar expressions can be derived. Each of these points is defined by its position with respect to the trocar as:

$$
\overrightarrow{T M}=: r_{M} \mathbf{z}_{2}
$$

The modeled task is the manipulation of a tissue. This action is modeled as a zero-length spring of unknown stiffness, attached between an arbitrary resting position $D_{R}$ and $D$. The force exerted by the spring on the tool is denoted $\mathbf{f}_{D}$.

In a typical surgical application, the surgeon applies a combination of torques and forces on the tool handle, creating the desired motion with minimal resulting forces on the trocar. However, solving the static equilibrium with these additional components would require additional instrumentation when used on an actual tool (i.e. requiring the knowledge of either the full user-applied wrench or the interaction forces at the trocar), we reduce the action of the user on the tool to a force $\mathbf{f}_{P}$ at $P$.

Finally the robot attached to $R$ is also capable of applying a force denoted $\mathbf{f}_{R}$ on the tool. As the model defined has no effect on a potential rotation along the $\left(T, \mathbf{z}_{2}\right)$ axis, this rotation has been purposely discarded from this work.

The linear velocity of the tool at point $M$ can be derived from the kinematic constraints as:

$$
\begin{aligned}
& \mathbf{v}_{M}=\dot{r}_{M} \mathbf{z}_{2}-r_{M} \mathbf{z}_{2} \times\left(\dot{\theta} \mathbf{y}_{0}+\dot{\varphi} \mathbf{x}_{1}\right)=\mathbf{J}_{M} \dot{q} \\
& \mathbf{J}_{M}=\left[\begin{array}{lll}
\mathbf{z}_{2} & r_{M} \mathbf{c}_{\varphi} \mathbf{x}_{2} & -r_{M} \mathbf{y}_{2}
\end{array}\right]
\end{aligned}
$$

where $c_{\varphi}$ is the cosine of the angle $\varphi, q=\left[\begin{array}{lll}r_{R} & \theta & \varphi\end{array}\right]^{T}$ is the configuration of the tool (note: $\forall M, \dot{r}_{R}=\dot{r}_{M}$ ) and $\mathbf{J}_{M}$ is the Jacobian of the system at the point $M$. As singularities occur when $r_{M}=0$ and when $\varphi= \pm \pi / 2$, we chose to define: $r_{P}<r_{R}<0<r_{D}$ and to limit $\theta$ and $\varphi$ to the range ]$-\pi / 2, \pi / 2[$.

Considering that the surgeon manipulates the tool with care and at low speed, and neglecting the action of gravity, we can assume that the tool is in static equilibrium submitted to the actions exerted by the user, the environment and potentially the actuation. Applying the principle of virtual power leads to the expression of the static equilibrium:

$$
\mathbf{J}_{P}^{T} \mathbf{f}_{P}+\mathbf{J}_{D}^{T} \mathbf{f}_{D}+\mathbf{J}_{R}^{T} \mathbf{f}_{R}=0 .
$$


As all the jacobian matrices are invertible, this expression can be reformulated so as to deduce the value of $\mathbf{f}_{P}$ as a function of $\mathbf{f}_{D}$ and $\mathbf{f}_{R}$. In base $B_{2}$ :

$$
{ }^{2} f_{P}=-\left[\begin{array}{ccc}
b_{D} & 0 & 0 \\
0 & b_{D} & 0 \\
0 & 0 & 1
\end{array}\right]{ }^{2} f_{D}-\left[\begin{array}{ccc}
b_{R} & 0 & 0 \\
0 & b_{R} & 0 \\
0 & 0 & 1
\end{array}\right]{ }^{2} f_{R}
$$

where $b_{M}:=r_{M} / r_{P}$ is the lever ratio between the points $M$ and $P$. Notice that $b_{M}$ is negative when $M$ and $P$ are on opposite sides of the trocar. Hence $b_{R}>0$ and $b_{D}<0$.

\section{FORCE FEEDBACK CONTROL}

As emphasized in Sec. I, both the displacement and the force are anisotropically amplified between the handle and the tip of a laparoscopic tool. Therefore, the perception of the distal displacement and force from the mechanical signals sensed at the surgeon's hand level suffer from distortion. However, the displacement is not only estimated by using the surgeons' touch perception, but also by using their visual perception. Literature provides evidences that the surgeons can compensate for the displacement amplification, as well as its inversion [17].

Our proposition is therefore to modify the stiffness perception by providing the user with a force feedback. Our aim is to apply a force to the handle in such a way that the total force experienced by the surgeon is equivalent to the force measured at the tool tip. To this aim, we formulate the desired behavior as:

$$
\mathbf{f}_{P}=\mathbf{C}_{m} \mathbf{f}_{D}
$$

where $\mathbf{C}_{m}$ is the relation between the desired user-felt forces at the proximal handle and the interaction force at the distal tool tip.

We propose to set:

$$
{ }^{2} C_{m}=\left[\begin{array}{ccc}
1 & 0 & 0 \\
0 & 1 & 0 \\
0 & 0 & -1
\end{array}\right],
$$

so that the force felt by the user (i.e. $-\mathbf{f}_{P}$ ) has the same magnitude as the force applied on the tool tip by the tissue, but with a direction that is compatible with the mechanical stability of the system. To this end, the force that the robot should apply on the tool is obtained by combining both the previous expressions with the equation (4):

$$
\mathbf{f}_{R}=-\mathbf{J}_{R}^{-T}\left(\mathbf{J}_{P}^{T} \mathbf{C}_{m}+\mathbf{J}_{D}^{T}\right) \mathbf{f}_{D}=: \mathbf{G f}_{D}
$$

where $\mathbf{G}$ is the operator relating $\mathbf{f}_{R}$ to $\mathbf{f}_{D}$ in the Cartesian space. Consequently, the expression of the forces that the robot should provide in order to balance the tool is

$$
\mathbf{G}=-\frac{r_{P}+r_{D}}{r_{R}}\left[\begin{array}{lll}
\mathbf{x}_{2} & \mathbf{y}_{2} & \mathbf{0}
\end{array}\right] \text {. }
$$

Note that with this compensation model, no compensation is required when the distal and proximal length are equal $\left(r_{P}=-r_{D} \Rightarrow \mathbf{f}_{R}=0\right)$.

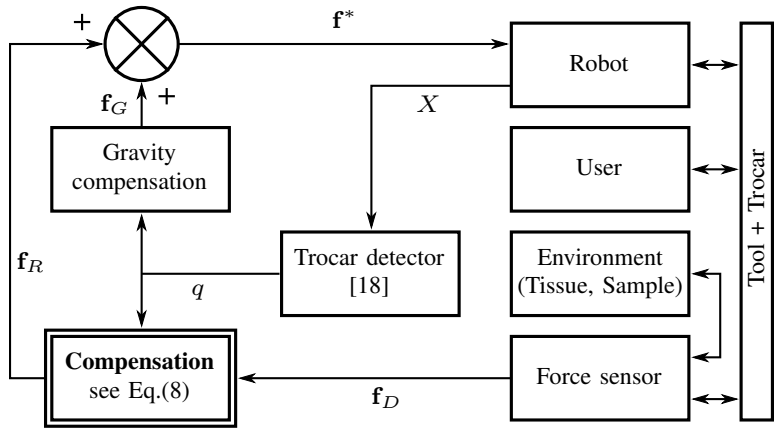

Fig. 4. Control scheme of the implemented system. $X$ denotes the configuration of the robot, combining the position of the wrist and the direction of the tool with respect to the robot base, and $\mathbf{f}^{*}$ is the force reference sent to the robot.

In practice, the controller implementing this strategy is presented Fig. 4.

The robot control input is a force $\mathbf{f}^{*}$, leaving the positioning task of the tool to the operator. A gravity compensation force (noted $\mathbf{f}_{G}$ ) was also added on top of the stiffness compensation force $\mathbf{f}_{R}$ given by Eq. (8) to improve the transparency of the tool. To compute the lever length $r_{P}$, $r_{R}$ and $r_{D}$, it is required to locate the trocar position with respect to the robot wrist. This is computed online using the automatic trocar detection algorithm described by Dong et al. [18]. This algorithm uses the position and orientation of the robot wrist to compute the fulcrum point using a least square optimization. This allows to robustly obtain the trocar position in any location of the robot workspace and to filter noisy joint position sensor signal without requiring additional external sensors, markers or cameras.

\section{IMPLEMENTATION}

In order to practically assess the results of this work, a prototype has been designed and implemented. The robot used for the test was a 3-DoF robot dedicated to collaborative or haptic tasks (Haption Virtuose 3D) with a passive 2-DoF spherical wrist at its extremity, instrumented to measure the orientation of the tool with respect to the robot base frame.

This robot was interfaced with a custom-designed instrumented tool that was composed of a 3D-printed rigid handle with a magnetic interface for fast (un)coupling with the robot and a $\varnothing 5 \times 300 \mathrm{~mm}$ aluminum rod, for a total tool length of $510 \mathrm{~mm}$.

This tool was terminated by a 6-DoF force/torque sensor (ATI Nano17-E SI-12-0.12) of which only the force components $\left(\mathbf{f}_{D}\right)$ were used for the control. On the measurement flange of the sensor was mounted a spherical indentation tip of $\varnothing 10 \mathrm{~mm}$ in order to allow a user to explore soft environments. Notice that this sensor would not be appropriate for a real implementation as it is too cumbersome and not sterilizable. In order to adapt this system for an actual clinical use, a specific force sensor dedicated to MIS should be used instead, as the one from Puangmali et al. [19]. 


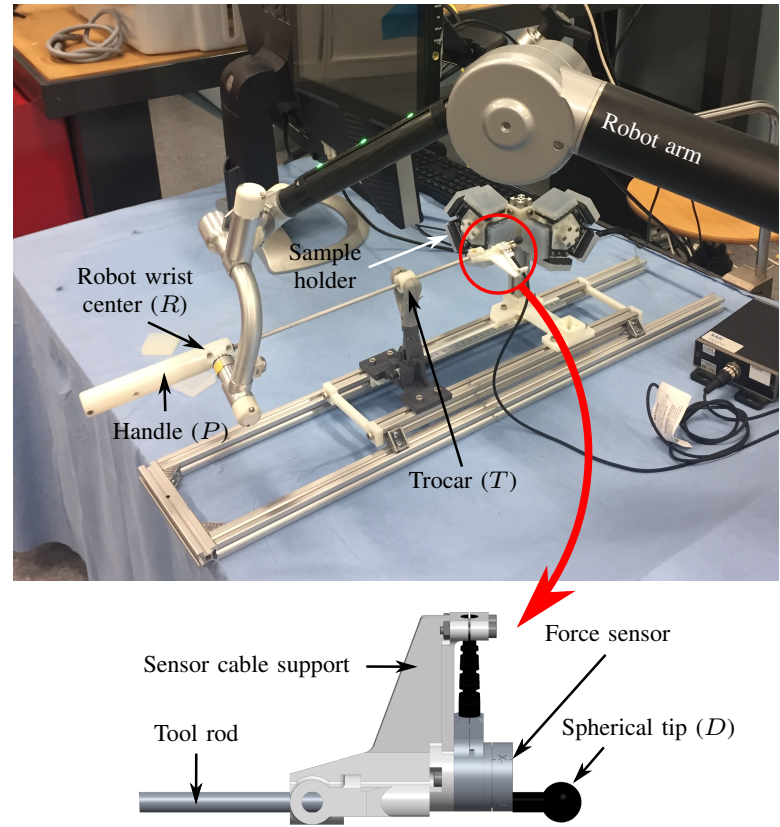

Fig. 5. Top: general view of the implementation. Bottom: CAD view showing details of the instrument tip.

The trocar constraint was enforced by a linear bearing assembled on a gimbal mount, allowing the tool to freely rotate in 3D and to translate along its longitudinal axis $\left(T, \mathbf{z}_{2}\right)$. The trocar and tool assembly were mounted on a frame, fixed with respect to the robot base, that also comprises two sample holders, each carrying a set of five samples made of silicon rubber with varied stiffness. Those samples can be presented to a user for palpation tasks, with one sample in the direction of insertion of the tool, and the other one perpendicular to it, allowing exploration by a swinging motion. The distance between the holder and the trocar can be adjusted in order to test the setting under several lever ratios. The rotation about the $\left(T, \mathbf{z}_{2}\right)$ axis was blocked, fixing the orientation of the tip (and force sensor) with respect to the robot wrist. The complete setup is shown on Fig. 5.

The control algorithm was running on a non-realtime OS (Microsoft Windows 10) at a sampling rate of $1 \mathrm{kHz}$. The dimensions of the tool used for the compensation computation were $R P=135 \mathrm{~mm}$ ( $P$ located at the back of the handle) and $R D=355 \mathrm{~mm}$ ( $D$ at the center of the spherical tip).

\section{EXPERIMENTAL EVALUATION}

\section{A. Experimental protocol}

An experimental protocol was designed to assess the efficiency of the force feedback controller, based on the evaluation proposed by Nisky et al. [4]. A two-alternative forced choice method (2AFC) allows to measure users' ability to compare samples of different stiffness. There are 5 samples of different stiffnesses, with the reference being the one in the center of the scale. Each comparison is always between the reference and another sample, including the reference itself. The two samples used in each trial were positioned in order to be explored using either a radial $[R]$ or tangential $[T]$ motion (as shown on Fig. 6). By performing this procedure with and without the force feedback compensation, the algorithm's efficiency can be evaluated.

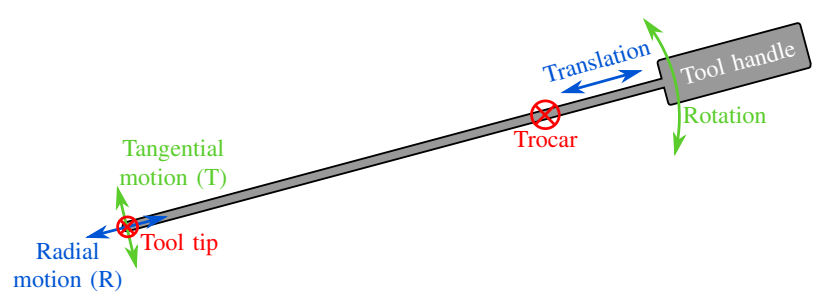

Fig. 6. Schematic representation of the possible tool motions.

The experiment had a $[2 \times 2]$ within-participants repeated measures design, with the factors being:

- COMPEnsation: the robot provides Compensation [C] or does Not provide Compensation [NC]; and,

- REFERENCE: the position of the reference sample is in the Radial [R] or Tangential [T] position.

The factors and their combination are sumarized in Table I.

\begin{tabular}{c|c|c} 
Condition & COMPENSATION & REFERENCE \\
\hline TNC & OFF & Tangential \\
TC & ON & Tangential \\
RNC & OFF & Radial \\
RC & ON & Radial \\
& TABLE I
\end{tabular}

EXPERIMENTAL CONDITIONS

1) Participants: We recruited 2 unpaid participants (1 female, ages 22-25) for the experiment. None of them had prior experience with laparoscopic surgery. Although performing this experiment with surgeons would give insights into the practical usefulness of the proposed method as either a gesture enhancer or a learning facilitator, our focus was on its raw impact on perception without introducing bias from experience.

2) Hardware and Software: Participants manipulated the system presented in Sec. III running an implementation of the compensation method. A dedicated software was developed to interface high-level functions of the robot control (namely brakes activation and toggling of the compensation method) with the experiment $\log$, to reduce experimental errors.

In order to limit the impact of visual cues [6] on stiffness perception, the direct observation of the samples was occluded by a screen. However, as cues for navigation where required, a video feed of the scene was shown on a touchenabled monitor in front of the participant where the surface of the samples was masked by overlaying black polygons (see Fig. 7).

Samples of variable stiffness were produced by the dilution of a silicone rubber (RTV EC00 Translucide, Esprit 


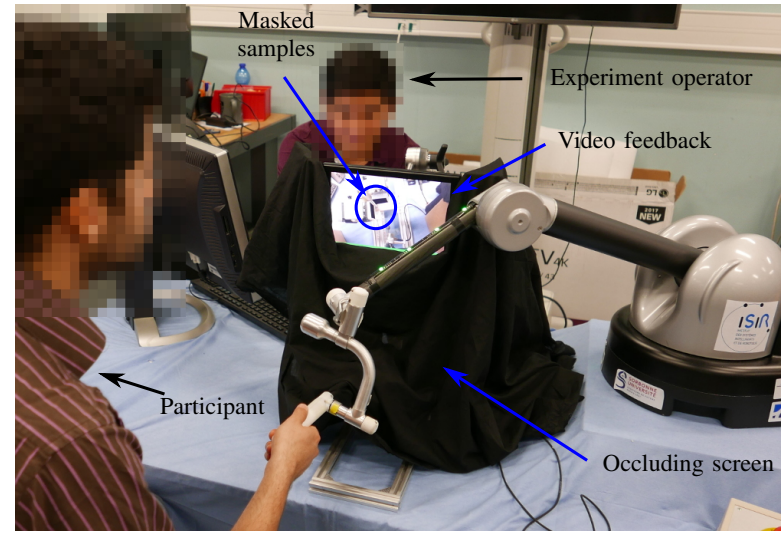

Fig. 7. General view of the experiment layout.

Composite) with varied levels of silicone thinner (DMF-50). Their stiffness was then assessed by indenting them with a $\varnothing 10 \mathrm{~mm}$ sphere, with the results shown in table II. Because of its intermediate stiffness in the available range, sample 3 was chosen as the reference sample (noted REF).

\begin{tabular}{c|c|c|c|c|c} 
Sample $(X)$ & 1 & 2 & REF & 4 & 5 \\
\hline Stiffness $\left(K_{X}\right)[\mathrm{N} / \mathrm{mm}]$ & 1.6 & 0.79 & 0.57 & 0.49 & 0.43 \\
\hline Stiff. ratio $\left(K_{X} / K_{R E F}\right)$ & 0.76 & 0.86 & 1 & 1.38 & 2.83 \\
TABLE II
\end{tabular}

SAMPLES AND ASSOCIATED STIMULUS LEVEL

In order to create bias in the perception, the fulcrum point of the tool was positionned to obtain a lever ratio value of $b_{D} \approx-0.34$.

3) Procedure: Participants manipulated a laparoscopic tool mockup protruding from the evaluation setup. They were given $20 \mathrm{~s}$ in order to freely explore both samples, with the instruction of avoiding the application of excessive force on the samples due to their low resilience. A warning was displayed when more than $8 \mathrm{~N}$ was measured on the sensor. After the time limit, the robot brakes were activated in order to stop further exploration of the samples.

Each of the conditions was tested with all samples, repeated 10 times in a fully randomized order for a total of 200 repetitions $(4$ conditions $\times 5$ samples $\times 10$ ).

The total experiment lasted approximately 2 hours, divided by a mandatory breaks at the end of each group of 40 repetitions in order to avoid fatigue.

4) Data Collection: For each trial, participants answered to the question Which sample is stiffer? by touching the corresponding sample on the monitor showing the video feed. The answer was logged along the conditions of the repetition in a dedicated file on the experiment computer.

5) Data Analysis: Participants answers are used to compute the probability of perceiving each sample stiffer than the reference. We fit a psychometric function to this probability as a function of the ratio between each sample and reference stiffness [20]. The curves average the data of both participants. Lastly, the PSE (Point of Subjective Equality) for each curve can be computed as the point where there is a $50 \%$ probability that a stimulus has a greater stiffness than the reference. The perception bias is obtained by measuring the difference between the PSE with a Point of Objective Equality (POE), i.e. the point where the $50 \%$ probability should be passed if no bias was present. Here the POE corresponds to the relative stiffness of 1 , where the sample presented has the same stiffness as the reference.

\section{B. Preliminary Results}

Figure 8 shows the psychometric curves of the four conditions, grouping both participants.

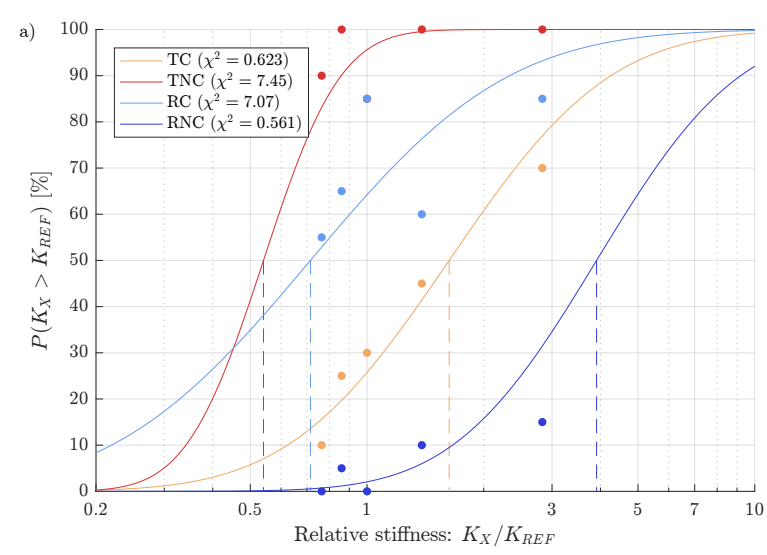

Fig. 8. Psychometric curves based on averaged results over individual experiments. The stiffness in abscissa is given as a ratio of the stimulus stiffness with respect to the reference stiffness. The $\chi^{2}$ test result for each fitted curve is given in the legend. Note: The measured value of $\mathrm{RC}$ and TNC are overlapping for $K_{X} / K_{R E F}=1$, hence the data point for TNC is not visible.

Each psychometric curve represents the estimation of the probability that a sample is perceived stiffer than the reference, as a function of the stiffness ratio. In such a plot, if the perception was unbiased, the curve would pass through the POE, of coordinates $[1 ; 50 \%]$; further, a highly reliable perception is characterized by a high slope around this point.

The results obtained tend to confirm the initial hypothesis: [TNC] (resp [RNC]) shows a negative (resp. positive) bias, thus this confirms the over-estimation of the stiffness of the tangential sample with respect to the radial one.

After compensation, the bias is pushed in the right direction for all cases but changed sign. This means that the compensation does occur, although somehow too strongly.

The obtained point cloud is relatively noisy, due to the low number of repetitions and participants (only 10 repetition per participant, giving 20 repetitions per data point), and as such the curves had a low goodness of fit (see fig. 8 for the results of the $\chi^{2}$ test). Additionally, most PSE used for quantifying the bias are located outside of the tested stimulus range, thus further limiting the validity of the computed bias. But although the results are not statistically valid and the data points do not cover the whole range of the curves, the 
curves show that the compensation may have an impact on the perception.

Sample manufacturing also introduced problems to the analysis. Although each sample's stiffness is tuned during the manufacturing by thinning the pre-cured silicone, the relation between thinner concentration and sample stiffness is complex. As such the samples obtained do not homogeneously cover the stimulus space, leading to a lower probability of correctly discriminating two samples with different stiffness levels.

Some options are currently being investigated in order to improve this experiments. The main flaw of the experiment is the number of repetition for each condition. This leads to statistical results with a low granularity, limiting the possibility of properly fitting the curves.

As the experiment time is already quite high, we propose to study the $[\mathrm{T}]$ and $[\mathrm{R}]$ factor in separate experiments, allowing twice the number of repetitions for each condition in the same amount of time. Each participant would then do either the $[\mathrm{R}]$ or the $[\mathrm{T}]$ experiment, not both.

Some work will also be directed in the improvement of the sample manufacturing: switching to a softer silicone may allow to work with lower levels of dilution, and will probably help in adjusting the obtained samples stiffness.

\section{Conclusion}

We present a method to compensate the distortion of the stiffness due to the fulcrum effect in laparoscopic surgery. The proposed compensation is based on a comanipulation robot that can apply forces to the laparoscopic instrument held by a surgeon. The system measures the force at the tool tip and compensates the distortion by applying a force to the handle.

The main advantage of this method is that it only requires as input the tool configuration and the force applied on its distal tip. This can be done regardless of the actuation method chosen for the implementation and of the intrinsic properties of the environment interacting with the tool.

With this system, a preliminary assessment work has been carried out, and its results have been discussed.

The proposed method is based on simplifying hypothesis in order to be implemented on tools with relatively low instrumentation requirements. Among them, the hypothesis that the trocar is stationary is most probably the less realistic. Indeed in real surgery the trocar point is not as clearly materialized and elastic interactions with the tool are possible. This will lead to a reduction of the forces felt by the user, and consequently an under-estimation of the distal stiffness.

Similar topics have been explored for positioning tasks with soft tissues interactions [21], and we believe that such developments could be adapted to the proposed method to overcome some of its limitations.

Some flaws have been observed in the experimental protocol, limiting its statistical significance. Future work will be focused on improving this protocol as discussed in order to carry out further experiments and prove the utility of this compensation method with novice users, and also if possible with expert surgeons.

\section{ACKNOWLEDGMENT}

This work was supported by French state funds managed by the ANR within the Investissements d'Avenir program (Labex CAMI - ANR-11-LABX-0004) and by the Région Grand Est.

\section{REFERENCES}

[1] C. R. Wagner, N. Stylopoulos, and R. D. Howe, "The role of force feedback in surgery: Analysis of blunt dissection," in Proceedings 10th Symposium on Haptic Interfaces for Virtual Environment and Teleoperator Systems. HAPTICS 2002, 2002, pp. 68-74.

[2] M. Zhou, S. Tse, A. Derevianko, D. *jones, S. Schwaitzberg, and C. Cao, "Effect of haptic feedback in laparoscopic surgery skill acquisition," Surgicl Endoscopy, vol. 26, no. 4, pp. 1128-1134, Apr. 2011.

[3] G. Picod, A. C. Jambon, D. Vinatier, and P. Dubois, "What can the operator actually feel when performing a laparoscopy?" Surgical Endoscopy, vol. 19, no. 1, pp. 95-100, Jan. 2005.

[4] I. Nisky, F. Huang, A. Milstein, C. M. Pugh, F. A. Mussa-ivaldi, and A. Karniel, "Perception of Stiffness in Laparoscopy - the Fulcrum Effect," Studies in health technology and informatics, vol. 173, pp. 313-319, 2012.

[5] W. M. B. Tiest and A. M. Kappers, "Kinaesthetic and cutaneous contributions to the perception of compressibility," in International Conference on Human Haptic Sensing and Touch Enabled Computer Applications. Springer, 2008, pp. 255-264.

[6] W. M. B. Tiest and A. M. L. Kappers, "Cues for Haptic Perception of Compliance," IEEE Transactions on Haptics, vol. 2, no. 4, pp. 189199, Oct. 2009.

[7] E. Fakhoury, P. R. Culmer, and B. Henson, "The effect of indentation force and displacement on visual perception of compliance," in 2015 IEEE World Haptics Conference (WHC), Jun. 2015, pp. 88-93.

[8] N. Gurari, K. J. Kuchenbecker, and A. M. Okamura, "Stiffness discrimination with visual and proprioceptive cues," in EuroHaptics Conference, 2009 and Symposium on Haptic Interfaces for Virtual Environment and Teleoperator Systems. World Haptics 2009. Third Joint, Mar. 2009, pp. 121-126.

[9] M. Kuschel, M. D. Luca, M. Buss, and R. L. Klatzky, "Combination and Integration in the Perception of Visual-Haptic Compliance Information," IEEE Transactions on Haptics, vol. 3, no. 4, pp. 234-244, Oct. 2010.

[10] R. H. Taylor, J. Funda, B. Eldridge, S. Gomory, K. Gruben, D. LaRose, M. Talamini, L. Kavoussi, and J. Anderson, "A telerobotic assistant for laparoscopic surgery," IEEE Engineering in Medicine and Biology Magazine, vol. 14, no. 3, pp. 279-288, May 1995.

[11] M. C. Cavusoglu, F. Tendick, M. Cohn, and S. S. Sastry, "A laparoscopic telesurgical workstation," IEEE Transactions on Robotics and Automation, vol. 15, no. 4, pp. 728-739, Aug. 1999.

[12] K. Tadano and K. Kawashima, "Development of a Master Slave System with Force Sensing Using Pneumatic Servo System for Laparoscopic Surgery," in Proceedings 2007 IEEE International Conference on Robotics and Automation, Apr. 2007, pp. 947-952.

[13] J. M. Sabater-Navarro, N. Garcia, J. Rodriguez, R. Morales, F. J. Badesa, and J. M. de Paco, "A simple and compact parallel robotic wrist for laparoscopy," in 2012 4th IEEE RAS EMBS International Conference on Biomedical Robotics and Biomechatronics (BioRob), Jun. 2012, pp. 835-840.

[14] Z. Zhang, H. Yu, and Z. Du, "Design and kinematic analysis of a parallel robot with Remote Center of Motion for Minimally Invasive Surgery," in 2015 IEEE International Conference on Mechatronics and Automation (ICMA), Aug. 2015, pp. 698-703.

[15] N. Zemiti, G. Morel, T. Ortmaier, and N. Bonnet, "Mechatronic Design of a New Robot for Force Control in Minimally Invasive Surgery," IEEE/ASME Transactions on Mechatronics, vol. 12, no. 2, pp. 143153, Apr. 2007. 
[16] L. Dong, "Assistance to laparoscopic surgery through comanipulation," Thesis, Université Pierre et Marie Curie - Paris VI, Dec. 2017. [Online]. Available: https://tel.archives-ouvertes.fr/tel-01721464

[17] I. R. Crothers, A. G. Gallagher, N. McClure, D. T. James, and J. McGuigan, "Experienced laparoscopic surgeons are automated to the "fulcrum effect": An ergonomic demonstration," Endoscopy, vol. 31, no. 5, pp. 365-369, Jun. 1999.

[18] L. Dong and G. Morel, "Robust trocar detection and localization during robot-assisted endoscopic surgery," in 2016 IEEE International Conference on Robotics and Automation (ICRA), May 2016, pp. 41094114.

[19] P. Puangmali, H. Liu, L. D. Seneviratne, P. Dasgupta, and K. Althoefer, "Miniature 3-axis distal force sensor for minimally invasive surgical palpation," IEEE/ASME Transactions on Mechatronics, vol. 17, no. 4, pp. 646-656, Aug 2012.

[20] F. A. Wichmann and N. J. Hill, "The psychometric function: I. fitting, sampling, and goodness of fit," Perception \& Psychophysics, vol. 63, no. 8, pp. 1293-1313, Nov 2001. [Online]. Available: https://doi.org/10.3758/BF03194544

[21] R. Chalard, D. Reversat, G. Morel, P. Mozer, and M.-A. Vitrani, "Precisely positioning the tip of an instrument inserted through an orifice with a free wrist robot: Application to prostate biopsies," International Journal of Computer Assisted Radiology and Surgery, vol. 13, no. 5, pp. 611-618, May 2018. 